\title{
Force and Accuracy Throws by Older Adults: II
}

\section{Kathleen Williams, Kathleen Haywood, and Ann VanSant}

\begin{abstract}
Older adults were tested to clarify findings of an earlier examination of movement responses to shifting task requirements (Williams et al., 1993). Eleven participants (average age $=77$ years) were evaluated on form and velocity as they performed overarm throws for force and accuracy. Significant gender and force-accuracy differences occurred for resultant velocity. Although no statistically significant differences occurred for force-accuracy comparisons of movement form, there were trends toward change in most movement components. Additionally, many individuals displayed change in one or more components as they shifted from force to accuracy throws. Results of this study point to the importance of examining developmental status and task requirements simultaneously.
\end{abstract}

Key Words: developmental sequences, aging, motor development

In an earlier investigation of older adults performing the overarm throw, Williams and colleagues (Williams, Haywood, \& VanSant, 1993) examined change in throws for force versus throws for accuracy. They reasoned that the shift from throwing a ball as fast or as hard as possible to throwing a ball at a target would alter velocity and movement patterns. This hypothesis was based on a model of constraints advanced by Newell (1986). In that model, Newell proposed that specific movement characteristics emerged as a result of the interaction between organismic, task, and environmental factors. Organismic factors were related to the organism itself, like strength and lever length. Task constraints were specific requirements of the stated task, like throwing for force or throwing for accuracy. Environmental constraints included current weather conditions or the effect of gravity on performance.

Langendorfer (1990) found changes in movement form in fourth-grade boys and adult men when they shifted from forceful to accurate throws, in support of Newell's model of task constraints. Male participants in that investigation used developmentally lower levels of throwing form when challenged to throw

Kathleen Williams is with the Department of Exercise and Sport Science, University of North Carolina at Greensboro, Greensboro, NC 27412-5001. Kathleen Haywood is with the Department of Physical Education, University of Missouri-St. Louis, St. Louis, MO 63121-4499. Ann VanSant is with the Division of Physical Therapy, Temple University, Philadelphia, PA 19122. 
accurately than when challenged to throw forcefully. Female participants made little change from throwing for force to throwing for accuracy. Langendorfer suggested that experience, skill level, or strength differences (differences in organism-task interactions) might account for gender differences. In another study, Roberton (1987) found declines in velocity scores for young children who threw under conditions of increasing unpredictability. Task requirements shifted from throwing for all-out force to throwing at a moving target. While there is no reason to expect that older adults' movement patterns or throwing velocities would change in different ways than those of children or young adults, there has been little systematic study of fundamental motor patterns in elders to document the course of change as it relates to aging or to task requirements, or their interaction.

Findings of the earlier study of older adults (Williams et al., 1993) were mixed. Men threw more slowly when an accuracy component was added to the task. Women used the same throwing velocity regardless of task constraints. When throwing form was evaluated, there were trends toward developmentally lower actions for accuracy throws for both men and women. None of these form differences reached statistical significance for either group.

The 1993 study was patterned after that of Langendorfer (1990). In both studies, the target used in the accuracy task was $10 \mathrm{~m}$ away from adult subjects. Subjects made forceful throws with the instruction to "throw as hard/far as you can" (no target was present). In response to that instruction, men threw using different velocities and tended to use lower developmental levels for accuracy throws, while women did not (Williams et al., 1993). This finding suggested that not all participants adapted their responses to the shift in task constraints. Results suggested that only the men shifted their responses in light of changing task requirements.

Two reasons were suggested to explain why women did not change their velocity or movement patterns. First, the distance of the target $(10 \mathrm{~m})$ may have required many women to use a forceful throw even when accuracy was emphasized verbally. Some women were barely able to reach the target "on the fly" with their throws. Thus, for those women, actual task requirements did not shift from force to accuracy as intended. Second, women used developmentally lower movement patterns than men for both types of throws. Williams and her colleagues (1993) suggested that men had more "options" to choose from as task requirements changed (Roberton, 1987). Women, who already threw using the developmentally lowest form, were unable to regress to a lower level.

The purpose of the present investigation was to examine further the issue of changing task constraints on the same group of older adults. Despite their diminishing numbers, it was useful to retest individuals who were long-term participants in an ongoing longitudinal investigation. This investigation remains one of the few longitudinal studies of fundamental motor skill performance by older adults.

Participants were tested in performing overarm throws for accuracy and force. In the current study, however, the target was moved closer to subjects and was made smaller, reducing the likelihood that any of the women would need their most forceful throw to reach the target. The change in target size also increased the contrast between force and accuracy conditions for all subjects. 


\section{Method}

\section{PARTICIPANTS}

Eleven participants (6 women, 5 men) were tested. Each had been tested previously as part of a longitudinal investigation of change in the overarm throw. Each participant also was involved in an Active Adult Program at the University of Missouri-St Louis. Women averaged 76.3 years of age ( $S D=$ 5.4 years, range $67-81$ years); men averaged 77.0 years $(S D=4.9$ years, range 69-82 years). Each participant read and signed an informed consent statement before testing.

\section{MOVEMENT TASK AND INSTRUMENTATION}

Participants were videotaped from the side, at a distance of $9.2 \mathrm{~m}$, with a Panasonic video camcorder (Model PV 330D). The camcorder had a high-speed, 1/1000-s shutter and recorded movement at approximately 30 fields per second. A comparison of the relatively slow speed of videotaped data with high-speed film suggested that movement actions can be analyzed with comparable levels of accuracy (Barrett, Williams, Bell, \& Allison, in press). Therefore, analyses conducted using standard videotaping techniques and speeds were considered adequate.

Testing took place indoors in a large field house. Subjects were tested individually and were allowed any amount of warm-up they desired before videotaping. Participants made five throws for maximum force and five throws for accuracy. Force trials always preceded accuracy trials since the forceful throws were part of the ongoing longitudinal study. When making forceful throws, subjects were instructed to throw tennis balls as hard as they could toward an unmarked wall over $36 \mathrm{~m}$ away. For accuracy throws, tennis balls were thrown at a 74-cm diameter, circular target, placed at shoulder height $6.5 \mathrm{~m}$ away. The target was an open circle, through which objects could be thrown.

\section{DATA REDUCTION AND ANALYSIS}

One hundred ten trials were available for analysis (55 each of the force and accuracy throws). Preliminary data reduction took two forms. One phase involved classifying all the trials according to their developmental level. The developmental categories used were hypothesized and validated (Table 1) by Roberton (Roberton \& Halverson, 1984) and by Haywood, Williams, and VanSant (1991). The first two authors viewed the trials using videodecks that enabled them to slow the speed of the action and to view movements field by field. Before we classified all the trials, intra- and interrater objectivity criteria of $85 \%$ exact agreement were met. One trial per subject was selected for this analysis. Intrarater agreement was $95 \%$ for the trunk and forearm actions, $100 \%$ for the foot, and $91 \%$ for humerus and backswing actions. Interrater agreement was $100 \%$ for the trunk and foot actions, $90 \%$ for the humerus, and $85 \%$ for the backswing and forearm actions.

The remaining data were then classified. Modal values were found for each movement component for each subject. Modes were analyzed for force and 
Table 1 Developmental Sequences for Movement Components of the Overarm Throw for Force

Level

Sequence description

Trunk action component

Level 1

Level 2

Level 3

No trunk action or forward-backward action

Upper trunk rotation or trunk "block" rotation

Differentiated rotation

Humerus action component

Level 1

Level 2

Level 3

Forearm action component

Level 1

Level 2

Level 3

Humerus oblique

Humerus aligned but independent

Humerus lags

No forearm lag

Forearm lag

Delayed lag

Foot action component

Level 1

Level 2

Level 3

No foot action

Ipsilateral foot action

Contralateral foot action, short step

Level 4

Contralateral foot action, long step

Preparatory backswing action

Level 1

Level 2

Level 2.5

Level 3

Level 3.5

Level 4

No backswing

Elbow and humeral flexion

Humeral lateral rotation

Circular, upward backswing

Shortcut circular, downward backswing

Circular, downward backswing

Note. Trunk, humerus, forearm, and foot actions are modified from Roberton and Halverson (1984). Backswing action is modified from Haywood et al. (1991). See those references for a full description of each category.

accuracy comparisons for the men's and women's data using Friedman two-way analyses of variance.

A video/computer motion analysis system (Peak Performance Inc., Englewood, CO) was used to obtain horizontal and vertical coordinates of the ball's position before and after release. The release point for each trial was identified through visual inspection of the videotape. Agreement regarding the frame of release was consistent, within \pm 1 frame. Then, two frames before and after the release point were digitized and stored electronically. Resultant release velocity was computed from these coordinates for each of the five velocity and accuracy trials. Velocity was analyzed using a 2 (gender) $\times 2$ (force vs. accuracy) $\times 5$ (trials) repeated-measures analysis of variance (ANOVA). 
Table 2 Gender and Force-Accuracy Differences (Means and Standard Deviations) in Resultant Overarm Throwing Velocities

\begin{tabular}{llr}
\hline & \multicolumn{2}{c}{ Resultant velocity $(\mathrm{m} / \mathrm{s})$} \\
\cline { 3 - 3 } & $M$ & $S D$ \\
\hline Gender & & \\
$\quad$ Female & 10.8 & 1.6 \\
$\quad$ Male & 14.2 & 2.5 \\
Throw & & \\
$\quad$ Force & 13.3 & 3.1 \\
Accuracy & 11.3 & 1.8 \\
& & \\
\hline
\end{tabular}

\section{Results}

\section{THROWING VELOCITY}

Resultant velocities were analyzed in a 2 (gender) $\times 2$ (force vs. accuracy) $\times 5$ (trials) repeated-measures ANOVA (Table 2). Significant main effects occurred for gender, $F(1,9)=18.97, p=.0018$, and throw type, $F(1,9)=8.68, p=.016$. The trials main effect was nonsignificant $(p>.05)$. No interactions reached significance. Men threw using faster velocities than women, and throws made for force traveled faster than throws made for accuracy.

\section{MOVEMENT COMPONENTS}

There were no statistically significant differences $(p>.05)$ for force-accuracy comparisons for any movement component for either men or women (Table 3). Average modes for each component suggested that both men and women tended to use a lower developmental level when throws were made for accuracy rather than for maximum force. Women moved toward a lower developmental level for all components except the forearm. Men moved toward a lower level of performance for all components.

While no significant group changes were documented for any movement component as task requirements shifted from force to accuracy, changes for individuals occurred. Modal categorizations for each component were compared for force and accuracy throws made by each participant. Three women were categorized as using lower level forearm and trunk actions for accuracy throws. Two women shifted to a lower level for humeral action, and 1 woman used a lower level foot action for her accuracy throw. For each movement component, 2 men shifted to a lower level pattern when throwing for accuracy. Different subjects shifted in their categorizations for different movement components, although several participants (men and women) were categorized at lower levels for more than a single component. 
Table 3 Average Modal Values (Means and Standard Deviations) of Developmental Levels in Overarm Throwing by Gender and Force-Accuracy Conditions

\begin{tabular}{|c|c|c|c|c|}
\hline \multirow[b]{2}{*}{ Movement component ${ }^{\mathrm{a}}$} & \multicolumn{2}{|c|}{ Force } & \multicolumn{2}{|c|}{ Accuracy } \\
\hline & $M$ & $S D$ & $M$ & $S D$ \\
\hline \multicolumn{5}{|l|}{ Women } \\
\hline Backswing (6) & 2.92 & 0.38 & 2.08 & 0.74 \\
\hline Forearm (3) & 1.00 & 0.00 & 1.00 & 0.00 \\
\hline Foot (4) & 3.17 & 0.41 & 3.00 & 0.00 \\
\hline Humerus (3) & 1.92 & 0.20 & 1.83 & 0.41 \\
\hline Trunk (3) & 2.00 & 0.00 & 1.50 & 0.55 \\
\hline \multicolumn{5}{|l|}{ Men } \\
\hline Backswing (6) & 3.50 & 0.71 & 3.10 & 0.90 \\
\hline Forearm (3) & 1.30 & 0.45 & 1.00 & 0.00 \\
\hline Foot (4) & 3.30 & 0.45 & 3.00 & 0.00 \\
\hline Humerus (3) & 2.20 & 0.45 & 1.90 & 0.22 \\
\hline Trunk (3) & 2.00 & 0.00 & 1.60 & 0.55 \\
\hline
\end{tabular}

Note. All differences are nonsignificant $(p>.05)$.

${ }^{a}$ Numbers indicate number of steps within the developmental sequence (see Table 1).

\section{Discussion}

Results of this investigation clarified those reported earlier by Williams and colleagues (1993). When the focus was shifted from making a forceful throw to making an accurate one, participants decreased the velocity of their throws. In contrast to the earlier investigation, in which only men demonstrated change, women in the present study also decreased their velocity when accuracy was emphasized. This finding suggests that moving the target closer and making it smaller resulted in a successful manipulation of task constraints. Additionally, while group differences were not detected for movement component measures, there was evidence that some individuals modified their throwing patterns when the task was changed from force to accuracy. There was no consistent pattern of change from one subject to another, however. Some individuals shifted to a developmentally lower pattern in several components, while others shifted in only a single component.

In the earlier investigation, Williams and colleagues (1993) offered two different explanations for their failure to find changes in women's throws. They suggested that actual task constraints (throwing distance) required women to make forceful throws, regardless of the stated purpose. Second, they hypothesized that the low developmental levels demonstrated by women made it impossible for them to regress further. Results of the present investigation point strongly to the latter hypothesis as an explanation of the current results. Placing a smaller target closer resulted in lowered throwing velocities, suggesting that the previously used distance did require all-out throws by most women. In contrast, 
many men and women were categorized at the lowest developmental levels for different components, especially humeral and forearm actions (Table 3), precluding additional regression.

Roberton (1987) and others (Halverson, 1966; Langendorfer, 1990) suggested that individuals who shift from one pattern to another under different task constraints make use of a range of movement options available to them. That is, participants who exhibit a certain movement pattern when all-out performance is necessary may use a developmentally lower pattern when the task requires a lower level of performance. For example, 3 women and 2 men in the present investigation used less advanced backswing actions when throwing for accuracy than for force. In contrast, participants who display the developmentally lowest form of an action have no developmentally lower categories open to them.

All of the women tested were categorized at Level 1 forearm actions, whether they threw for force or for accuracy. Therefore, any change in these participants' performance would not be detected by the movement components evaluated in this investigation. For those individuals, change could be observed only as lowered throwing velocity (as observed in this investigation) or as quantitative change within their Level 1 forearm action, like a smaller range of forward motion. Similar quantitative shifts could occur within other movement components as well. For example, any degree of blocked (upper and lower trunk together) trunk rotation places a thrower at Level 2 in the trunk component sequence. A participant could use less trunk rotation when throwing for accuracy than for force. Despite changes in range of motion, both types of throws would be placed at Level 2 . The inability of these developmental sequences to distinguish between different amounts of quantitative change in form (like amount of trunk rotation) is a limitation inherent in these measures of qualitative change.

The inability to measure within-level change was a limitation of this investigation (and any investigation that relies on categorizations of qualitative change). It is important to recognize that individuals demonstrated change in movement form in response to changing task constraints, although no significant group change was detected. The small number of subjects available to continue their participation in the longitudinal aspects of this investigation resulted in insufficient power to detect any change that may have occurred. Thus, it is important to acknowledge the change observed in individual participants. Although velocity emerged as a more sensitive measure of change, clearly individuals were influenced by the manipulation performed in this investigation. Roberton (1987) similarly found throwing velocity to be a more sensitive measure of change than movement form in her study of changing task constraints in young children.

A limited range of task constraints was tested in this and the previous investigation (Williams et al., 1993). The primary manipulation involved a modification of throwing distance within the accuracy throw condition. It seems logical that a clearer change in velocity or a change in more movement components might be observed if additional conditions were added. That is, clearer evidence of change might have been observed if participants had thrown at targets arranged at a variety of different distances. The number of conditions tested was limited by the age and stamina of the individuals tested. Subjects averaged 77 years; several were in their $80 \mathrm{~s}$. Due to the advanced age of the participants, we decided to minimize fatigue and injury potential by limiting the number of experimental conditions. 
Results of this investigation add to a growing body of information about the interaction between task requirements and movement process and product. As demonstrated in this and the previous study (Williams et al., 1993), a shift in task requirements changes the way the movement is performed. For example, an individual could throw a ball using a lagging forearm, a differentiated trunk, and a long contralateral step, resulting in a relatively forceful outcome. If the task is to toss a ball to a partner 10 feet away, however, the force generated by such a throw may injure or alienate one's partner. In this situation, all-out force is unnecessary. Instead, the throw must be modified so that it is manageable for the partner; using little or no forearm lag, some block rotation, and a short step would be more appropriate. When evaluating this throw, an uninformed observer might ignore task context and incorrectly conclude that the thrower was at a low level of development. A correct conclusion would be that the thrower appropriately modified the action for the context. Clearly, movement process and outcome must be evaluated in light of specific task constraints.

In summary, the smaller, closer target used in this investigation elicited more change from the "forceful" throw than occurred in the earlier investigation. The older adults adapted their movement responses to the task requirements but did so within the limitations of their current developmental status. Those with less advanced patterns had fewer options for change. Those demonstrating more advanced patterns for forceful throws had more options and chose from among them as they shifted to accuracy throws. Results of this investigation point to the importance of examining developmental status and task requirements simultaneously.

\section{References}

Barrett, K., Williams, K., Bell, R., \& Allison, P. (in press). The degree of observer agreement on an intraskill developmental sequence using videotape and high speed film. In J.E. Clark \& J. Humphrey (Eds.), Advances in motor development research (Vol. 4). New York: AMS Press.

Halverson, L.E. (1966). Development of motor patterns in young children. Quest, 6, 44-53. Haywood, K., Williams, K., \& VanSant, A. (1991). Qualitative assessment of the backswing in older adult throwing. Research Quarterly for Exercise and Sport, 62, 340-343.

Langendorfer, S. (1990). Motor-task goal as a constraint on developmental status. In J. Clark \& J. Humphrey (Eds.), Advances in motor development research (Vol. 3, pp. 16-28). New York: AMS Press.

Newell, K.M. (1986). Constraints on the development of coordination. In M.G. Wade \& H.T.A. Whiting (Eds.), Motor development in children: Aspects of coordination and control (pp. 341-361). Amsterdam: Martinus Nijhoff.

Roberton, M.A. (1987). Developmental level as a function of the immediate environment. In J. Clark \& J. Humphrey (Eds.), Advances in motor development research (Vol. 1, pp. 1-16). New York: AMS Press.

Roberton, M.A., \& Halverson, L.E. (1984). Developing children-Their changing movement. Philadelphia: Lea \& Febiger. 
Williams, K., Haywood, K.M., \& VanSant, A. (1993). Force and accuracy throws by older adult performers. Journal of Aging and Physical Activity, 1, 2-12.

\section{Author Note}

The authors thank the participants in this investigation for their continued interest and support of the project. We thank two anonymous reviewers for their thoughtful comments on an earlier version of this manuscript. 